\title{
What the Internist Should Know about Thrombotic Microangiopathies
}

\author{
Marjan Alidoost*, Attiya Haroon, Varsha Gupta, Arif Asif, Mohammad A. Hossain \\ Department of Medicine, JSUMC, Neptune, NJ, USA \\ Email: *marjan.alidoost@hackensackmeridian.org
}

How to cite this paper: Alidoost, M., Haroon, A., Gupta, V., Asif, A. and Hossain, M.A. (2019) What the Internist Should Know about Thrombotic Microangiopathies. International Journal of Clinical Medicine, 10, 651-661.

https://doi.org/10.4236/ijcm.2019.1012054

Received: November 18, 2019

Accepted: December 16, 2019

Published: December 19, 2019

Copyright $\odot 2019$ by author(s) and Scientific Research Publishing Inc. This work is licensed under the Creative Commons Attribution International License (CC BY 4.0).

http://creativecommons.org/licenses/by/4.0/

\begin{abstract}
Thrombotic microangiopathy (TMA) is a group of diseases that are lifethreatening and can lead to end organ damage (EOD) due to ischemia caused by microthrombi in capillaries and arterioles. TMAs can affect any organ system but usually affect the kidney, intestines, and nervous system. The triad of TMA is Coombs-negative hemolytic anemia with schistocytes seen on peripheral smear, thrombocytopenia (platelets under 150,000 or a decrease of $25 \%$ or more from baseline), and evidence of ischemic EOD. Primary TMAs include Thrombotic Thrombocytopenic Purpura (TTP), Hemolytic Uremic Syndrome (HUS) and atypical HUS (aHUS). Pathophysiologically, all of these diseases are caused by aggregation of von Willebrand Factor (vWF) multimers, via different mechanisms, which eventually leads to thrombus formation. TTP and aHUS benefit from plasma exchange (PEX), whereas HUS is treated symptomatically. Urgent recognition with timely treatment is crucial to managing these potentially life-threatening conditions.
\end{abstract}

\section{Keywords}

TMA, aHUS, HUS, TTP

\section{Introduction}

Thrombotic microangiopathy (TMA) is a group of diseases that are life-threatening and can lead to end organ damage due to ischemia caused by microthrombi in capillaries and arterioles [1]. TMAs can affect any organ system but usually affect the kidney, intestines, and nervous system [2]. The triad of TMA is Microangiopathic Hemolytic Anemia (MAHA), defined as Coombs-negative hemolytic anemia with schistocytes seen on peripheral smear, thrombocytopenia (platelets under 150,000 or a decrease of $25 \%$ or more from baseline), and evidence of ischemic EOD [2]. 
Although characteristic symptoms vary between the various TMAs, renal and neurological symptoms remain particularly prominent [3]. TMAs can affect any organ; however, renal and neurological symptoms are particularly prominent [3]. In fact, $12 \%$ of patients with typical Hemolytic Uremic Syndrome (HUS) develop ESRD or death, and among patients who survive, 25\% develop some type of renal sequalae [4]. It has been estimated that $12 \%$ of patients with Thrombotic Thrombocytopenic Purpura (TTP) present with neurological abnormalities [4]. Since TMAs can affect any organ it is also possible to present with primary symptoms affecting other organs, such as cardiac ischemia. In fact, the first patient to be diagnosed with a TMA by Moschcowitz in 1924 presented with hemiparesis, died with heart failure and autopsy showed hyaline thrombi in most of her organs [3]. Although it is not necessary to have all of the classical symptoms to diagnose TMA it is important to have the clinical suspicion to test for it.

Symptoms that suggest TMAs include typical anemia symptoms (such as fatigue, dizziness, shortness of breath), increased bleeding and bruises (due tothrombocytopenia), altered mental status, headache and seizures (due to damage to blood vessels in the brain), decreasing urine output and lower extremity edema (due to damage to blood vessels in the kidney). On a histopathological level the damage caused by TMAs arises from platelet von Willebrand Factor (vWF) multimer aggregation with subsequent edema of the endothelium which causes fragmentation of erythrocytes [5]. However, the mechanism by which that happens differs greatly between the different TMAs. Since TMAs have a variety of different causes, they therefore have a variety of different treatments, ranging from supportive treatment to monoclonal antibodies such as eculizumab. It is therefore imperative for a clinician to not only be able to suspect a TMA, but also to be able to properly identify the various etiologies of TMAs [3]. Since there are so many etiologies of TMAs, it is beneficial to categorize TMAs into different subtypes.

A useful way for the clinician to categorize TMA is primary versus secondary, with primary syndromes being symptoms caused by the main disease process and secondary being those resulting from a systemic disease. Primary TMAs, which this article will focus on, include TTP, HUS, and atypical Hemolytic Uremic Syndrome (aHUS, also called complement-mediated TMAs), drug induced TMAs (most classically due to quinine and VEGF inhibitors) [2] and, rarely, vitamin B12 deficiency [6]. Secondary TMAs include pregnancy related including Hemolysis Elevated Liver Enzymes Low Platelet (HELLP) syndrome, systemic infections [7], Disseminated Intravascular Coagulation (DIC), malignancy induced TMAs, Scleroderma Renal Crisis, and lupus-associated TMAs, and malignant hypertension related TMAs [3]. A more comprehensive list of miscellaneous causes of TMAs can be seen in Table 1 .

Useful methods to help differentiate between primary and secondary TMAs are taking a thorough history which screens for causes of secondary TMAs. As a general primary TMAs usually involved kidney injury and have acute onset of several days duration. Exceptions to this rule are that 1) TTP commonly does 
Table 1. There are many causes of TMAs beyond TTP, HUS, and aHUS, which are the focus of this paper. When a patient presents with a possible TMA all differentials, including the above, should be considered.

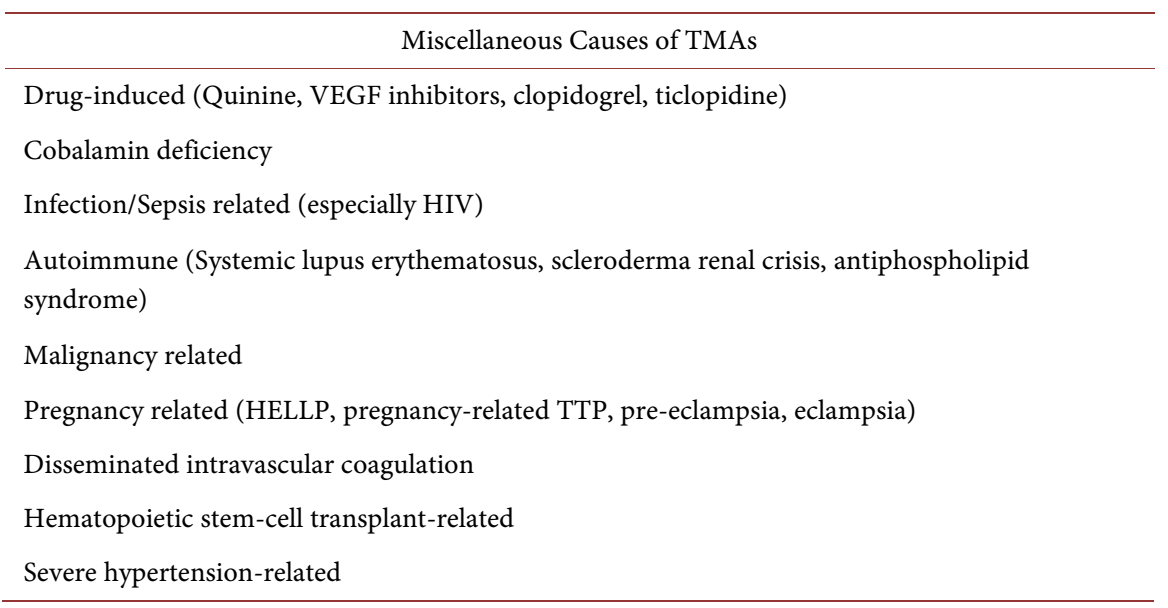

not present with kidney injury [1], and 2) that drug mediated TMAs tend to take place immediately over a couple hours duration. With the information provided below in mind the clinician should be confident in their ability to evaluate for primary TMAs.

\section{Thrombotic Thrombocytopenic Purpura}

TTP can occur in acquired or hereditary forms, although the acquired form is more common, especially in adults. TTP arises when there is a defect in the A Disintegrin and Metalloprotease with a Thrombospondin type 1 motif, member 13 (ADAMTS13), a metalloprotease that degrades vWF multimers [5]. In hereditary TTP (Upshaw-Shulman syndrome) ADAMTS13 has mutations which result in a non-functional enzyme. The hereditary form presents early in childhood-occasionally in pregnancy-and has a worse prognosis than acquired TTP [8]. In acquired TTP, which we will focus on more in this article, the patient develops antibodies against ADAMTS13 leading to decreased function of the metalloprotease, as illustrated in Figure 1 [9]. Acquired TTP tends to affect females and African Americans disproportionately [8]. TTP can present with a myriad of symptoms but the classic pentad of TTP is MAHA, thrombocytopenia, fever, renal abnormalities, and neurological abnormalities [5]. Neurological symptoms range from subtle changes in mentation to stupor and coma. There is considerable overlap between the symptoms of TTP and other TMAs although there are some key differences that can be observed on a pathophysiological level.

Pathophysiologically, the thrombi of TTP have higher levels of platelets and less fibrin than those of HUS. Additionally, TTP tends to have more widespread thrombi, leading to a more diverse array of symptoms than HUS, which usually has thrombi in the kidneys and the main symptom of which is kidney failure [8]. Of note, TTP tends to spare the lungs [10] and does not cause renal failure even 


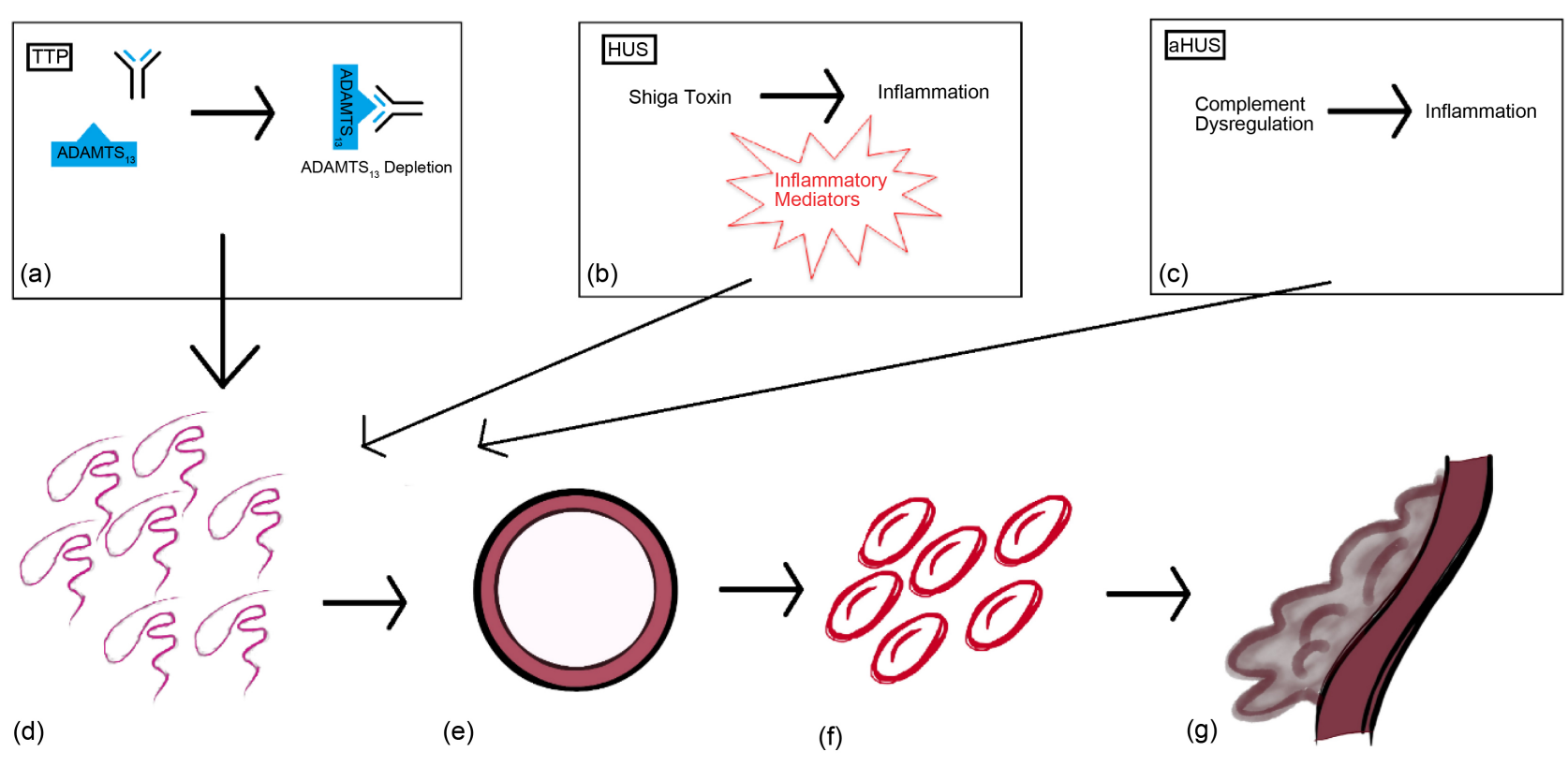

Figure 1. A basic schema of the pathophysiology of primary TMAs. (a) Shows autoantibody formation causing ADAMTS13 depletion in TTP; (b) Shows Shiga toxin causing release of pro-inflammatory mediators and inducing release of vWF as occurs in HUS; (c) Shows cytokine dysregulation leading to inflammation. (d) Shows vWF multimer aggregation; (e) Shows the resulting endothelial edema; (f) Shows erythrocyte aggregation; (g) Shows the eventual resulting thrombus.

though microthrombi are observed in the kidneys. The presenting clinical features of patients with TTP were analyzed by the Oklahoma TTP-HUS Registry, which was a 20 -year cohort study of 382 consecutive patients who were diagnosed with TTP or HUS [11]. Of those 382 patients, 18 were found to have severe ADAMTS13 deficiency, defined as ADAMTS13 activity under 5\%, and of those 18 patients, 10 had normal renal function [11].

To most reliably differentiate between TTP and other TMAs can be done via the ADAMTS13 assay which detects ADAMTS13 enzyme activity. ADAMTS13 activity under $10 \%$ is defined as severe deficiency and is often used as a benchmark to diagnose TTP in the right clinical setting [11]. However, the ADAMTS13 assay can take a long time to come back, and therefore cannot be used to make clinical decisions in real time. To more quickly identify which patients have TTP, defined as ADAMTS13 deficiency, the clinician can use the PLASMIC score, illustrated in Table 2. The PLASMIC score was developed in 2017 in a cohort study and was done using patients that presented to three large academic medical centers in Boston [12]. The PLASMIC score is shown in Table 2, with a score of 5/7 denoting intermediate risk and a score of 6-7/7 denoting high risk for TTP [12]. Patients with presumptive TTP should be treated for TTP and treatment should not be delayed by waiting for an ADAMTS13 assay.

It is vital to start Plasma Exchange (PEX), accompanied by corticosteroids, in patients with TTP, or suspected TTP, since PEX has been shown to cause remission in $70 \%-90 \%$ of patients [13]. PEX works by removing the auto-antibodies to ADAMTS13, and, along with steroids, is considered standard of care for patients with TTP [3]. Glucocorticoids are thought to decrease production of the 
Table 2. The PLASMIC score is a clinical scoring system that the clinician can use to help risk stratify patients for their risk of having TTP. The PLASMIC score is out of 7, with a score of 0 - 4 denoting low probability, 5 denoting intermediate probability, and 6 - 7 denoting high probability.

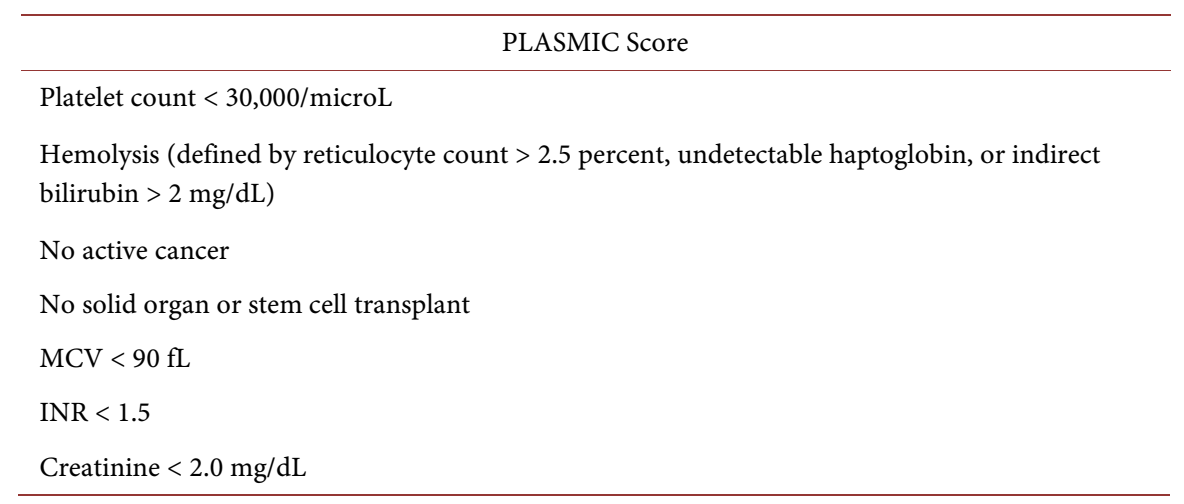

autoantibody against ADAMTS13 as they are immunosuppressive agents. PEX has drastically improved outcomes for patients with TTP, with untreated TTP having a mortality as high as $90 \%$ [14]. Relapse is a significant concern for many patients with rates of recurrence as high as 50\% [15]. The only predictors for relapse that have been identified thus far are severe ADAMTS13 deficiency (under $10 \%)$ and the male sex [16]. Currently, patients are not given preventative treatment for relapse, and are monitored outpatient and given PEX and corticosteroids. There have been studies indicating that Rituxan, when administered to high risk patients, can reduce risk of relapse [17]. These studies were limited, in that they were retrospective, but given that Rituxan is generally a well-tolerated drug, it is a good discussion to have with Hematology whether or not to add Rituxan [17].

\section{Hemolytic Uremic Syndrome}

HUS is a form of TMA characterized by a triad of thrombocytopenia, microangiopathic hemolytic anemia and acute kidney injury. The name HUS was first coined in 1955 [3]. It encompasses a group of disorders including the typical HUS and the atypical HUS. Typical HUS affects predominantly the kidneys [3]. It is most commonly associated with gastrointestinal infection with Shiga toxin-producing Entero-Hemorrhagic Escherichia coli (EHEC) strains. EHEC was first associated with hemorrhagic colitis during an outbreak in the USA in 1982 [18]. Source of infection is usually intake of contaminated food such as undercooked meat, vegetables, unpasteurized milk products and also contaminated water [19]. Many strains of E. coli have been reported to cause HUS which includes E. coli 026, O13, 0111, O14 [19]. Shiga-toxin producing E. coli (STEC) expressing somatic $(\mathrm{O})$ antigen 157 and flagellar $(\mathrm{H})$ antigen 7 are the serotype most frequently isolated from infected individual [20]. The strain is commonly known as STEC O157:H7.

HUS may develop within 2 - 12 days after the gastrointestinal phase, mani- 
festing most commonly as bloody diarrhea [20]. Diagnosis of HUS requires two important steps. Firstly, laboratory tests to establish mechanical hemolytic anemia, thrombocytopenia and kidney injury. Secondly, microbiological tests to identify causative organism which includes stool culture for STEC, PCR for EHEC genes or ELISA for free Shiga toxin [21]. EHEC strains colonize the intestine after ingestion and release Shiga toxin. After injury to intestinal endothelium, toxin gains access to circulation, leading to platelet and leukocytes activation. The circulating toxins also play a role in damaging the glomerular endothelial cells. The combination of activated platelets and damaged endothelium induces thrombosis [19] [20].

The best way to prevent HUS is to prevent primary gastrointestinal infection. Once there is evidence of infection, fluid administration in the gastrointestinal phase reduces the risk of developing HUS. The use of antibiotics and anti-motility drugs has been associated with increased risk of developing HUS. Antibiotics use can lead to toxin release secondary to antibiotic induced bacterial membrane injury [21]. Notably, elderly and young children are reported to have an increased risk of developing HUS [21].

Treatment for HUS is generally supportive, with the mainstay of treatment being aggressive hydration and avoiding nephrotoxic medications to prevent kidney damage, however it is common for patients to require temporary dialysis [3]. Correcting other concurrent issues, such as electrolyte disturbances and acidosis, is also standard [3]. Patients with HUS typically make a full renal recovery, however for patients who do not respond to conservative measures renal transplantation may be indicated [3]. PEX has been used for patients with HUS but the benefits are not established [3]. For the anemia accompanying HUS, there are no unique guidelines-as with patients with anemia of other causes, patients should get a transfusion if their hemoglobin is below 7, unless they have acute coronary syndrome in which case their goal hemoglobin is 8 .

Many patients with EHEC associated HUS have been associated with full recovery [3]. Signs of poor prognosis include presence of neurological symptoms, high neutrophil counts, low platelet counts and duration of anuria [21]. New agents that neutralize the effect of Shiga toxin needs to be determined [3]. As well as measures to prevent entero-hemorrhagic infections should be the primary focus to avoid typical HUS.

\section{Atypical HUS}

Atypical hemolytic-uremic syndrome (aHUS) belongs to class of thrombotic microangiopathy and is characterized by endothelial injury and manifests as vascular thrombosis with severe organ dysfunction [22]. An urgent recognition with timely treatment, especially in modern medicine era with the proven efficacy of terminal complement inhibitors in treatment, is crucial to managing this life-threatening condition. Initial presentation of aHUS and severity of condition depends upon the pattern of organ dysfunction. Clinical features of AHUS are 
overlapping with TTP. Vascular thrombosis in aHUS affects kidney, brain, lung, gastrointestinal tract, however unlike TTP, $60 \%$ of the aHUS patients progressed to end-stage renal disease (ESRD) [23] [24]. It has also been suggested that a serum creatinine level of $>150$ to $200 \mu \mathrm{mol} \cdot \mathrm{L}^{-1}$ or a platelet count of $>30 \times 10^{9} \mathrm{~L}^{-1}$ "almost eliminates" a diagnosis of TTP [24].

aHUS is caused by genetic or acquired uncontrolled activation of alternate complement pathway in $40 \%-60 \%$ of patients [25]. Complement mediated endothelial cell damage is principle pathophysiology of aHUS which subsequently leads to formation of microthrombi more commonly glomeruli microthrombi. Genetic mutation in AHUS involves either loss of function mutations within membrane co-factor protein (CD46), complement factor $\mathrm{H}(\mathrm{CFH})$ and factor I (CFI), and autoantibodies to the factor $\mathrm{H}(\mathrm{FH})$ and factor I (FI) proteins. Or gain-of-function mutations within complement factor B (CFB) and C3 [26]. Raina et al. in a recent review reported that over activation of complement pathways occurs due to either the production of $\mathrm{FH}$ autoantibodies or due to genetic complement protein mutations such as FH, FI, FB, C3, and thrombomodulin [27] [28].

Granular C3 deposits in the glomeruli and arterioles during the intensive phase of the disease, leading to activation of complement and local C3 utilization activation of the Membrane Attack Complex (MAC) (C5b-9) results in microvascular thrombosis, especially within the kidneys. The $\mathrm{C} 3$ convertase of the classical and lectin complement pathways is composed of $\mathrm{C} 2$ and $\mathrm{C} 4$ fragments; however, the $\mathrm{C} 3$ convertase of the alternative pathway splits $\mathrm{C} 3$, but has no effect on C4 Because low serum C3 levels mirror complement activation, reduced levels of C3 and normal C4 is characteristic of aHUS However, not all patients with aHUS show hypocomplementemia [29].

aHUS can present at any age, as systemic disease and acute in $20 \%$ of cases [27]. Sign and symptoms essentially depend upon the extent of microthrombi and involvement of various organs. Kidney microvascular injury in aHUS could manifest as hematuria, proteinuria, hypertension, azotemia and volume overload.

aHUS is diagnosed according to criteria published by the UK aHUS Rare Diseases Group and European guidelines including the presence of both TMA and acute kidney injury without ADAMTS-13 deficiency or inhibitors [25].

Important diagnostic elements include thrombocytopenia (platelet count $<150,000 / \mathrm{mcL}$ or $25 \%$ decrease from baseline), microangiopathic hemolytic anemia (schistocytes on blood film, elevated lactate dehydrogenase, decreased haptoglobin, decreased hemoglobin) and target organ injury (elevated blood urea nitrogen and creatinine, abnormal liver function tests, elevated pancreatic enzyme levels, stroke, myocardial infarction etc.) [30].

Laboratory investigations such as Shiga-toxin test and ADAMTS13 activity can be very helpful in distinguishing HUS, TTP and aHUS from one another. A deficiency of ADAMTS13 (less than 5\% of normal activity) points to the diagnosis 


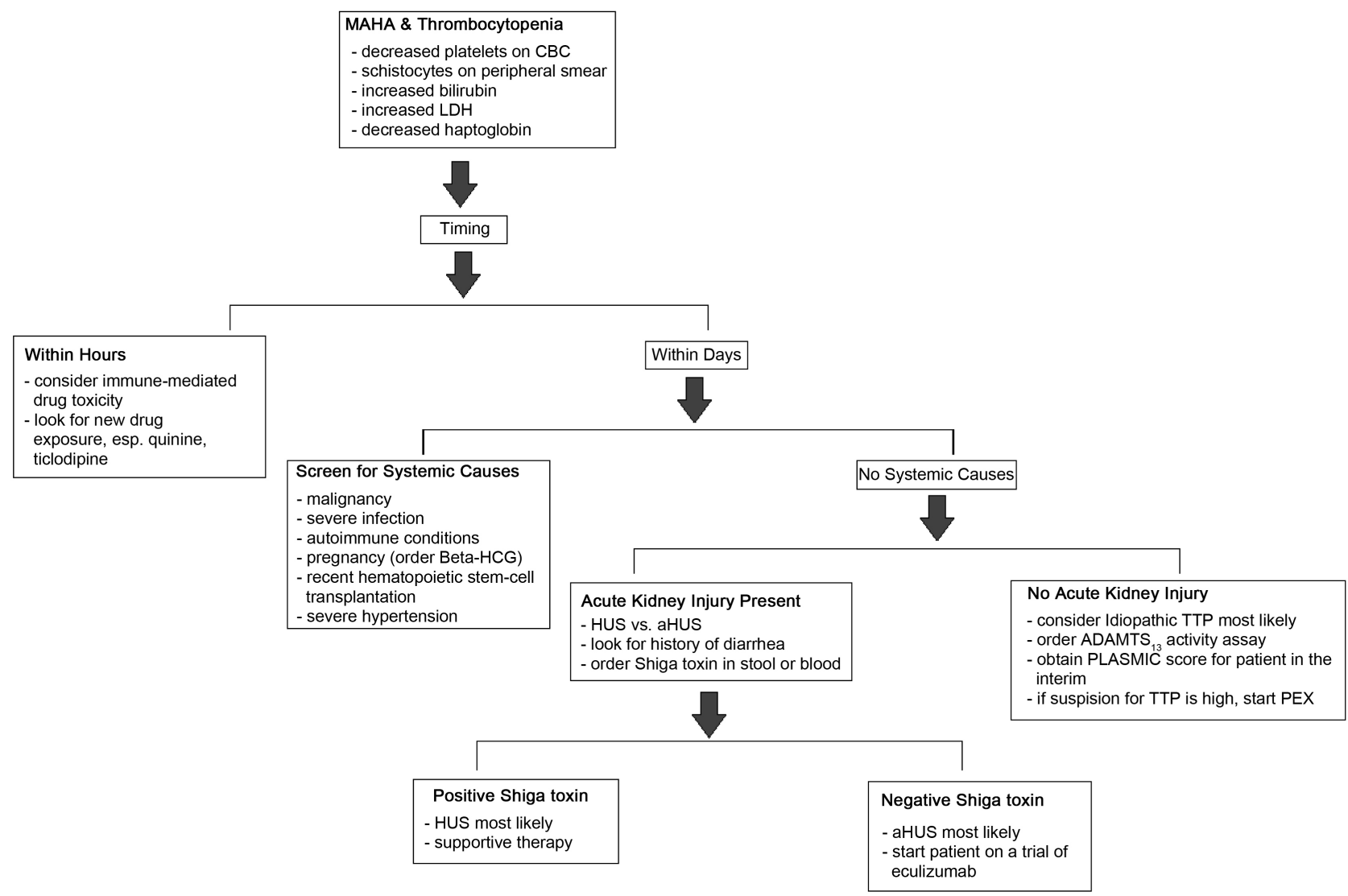

Figure 2. Algorithm for the evaluation of TMAs in the adult patient.

of TTP while the presence of Shiga toxin indicates STEC-HUS [31]. Normal ADAMTS13 activity and absence of Shiga toxin help establish the diagnosis of aHUS in patients presenting with thrombotic microangiopathy.

PEX is also indicated as standard treatment of aHUS and has significantly decreased mortality from $50 \%$ to $25 \%$ [32]. PEX is thought to improve outcomes in patients with aHUS by removing complement regulatory proteins [3]. However, even though PEX has helped outcomes in aHUS, many patients do not respond. For these patients, treatment with Eculizumab, has been approved by the Food and Drug Administration [32]. Eculizumab is a humanized monoclonal antibody which inhibits the complement pathway by blocking MAC formation by preventing cleavage of C5. Case reports have shown benefit of Eculizumab treatment, although this has not yet been shown in large randomized control trial studies [32].

\section{Conclusion}

There is certainly nuance to correctly diagnose TMAs. However, an organized framework to approach TMAs is useful, and our suggested algorithm is in Figure 2. The first step to diagnose TMA is to take a good history and physical, including timing and patient demographics, which can often on its own lead the clinician to the proper diagnosis. A good initial assessment of a patient's com- 
plaint can help exclude systemic disorders which can present similarly to TMAs, such as systemic infection mimicking TTP. It is important for the clinician to confirm MAHA on a peripheral smear and thrombocytopenia on CBC. Of note it is also useful to order ADAMSTS13 first since HUS and TTP have such different treatment modalities and TTP mortality is drastically changed by early treatment. TTP and aHUS patients usually benefit from plasmapheresis whereas HUS patients do not. Patients with HUS can be treated with supportive care and monitoring while patients with refractory aHUS may benefit from Eculizumab therapy which is an exciting area of research.

\section{Conflicts of Interest}

The authors declare no conflicts of interest regarding the publication of this paper.

\section{References}

[1] Brocklebank, V., et al. (2017) Thrombotic Microangiopathy and the Kidney. Clinical Journal of the American Society of Nephrology, 13, 300-317. https://doi.org/10.2215/CJN.00620117

[2] Bommer, M., et al. (2018) The Differential Diagnosis and Treatment of Thrombotic Microangiopathies. Deutsches Arzteblatt International, 115, 327-334. https://doi.org/10.3238/arztebl.2018.0327

[3] George, J.N. and Nester, C.M. (2014) Syndromes of Thrombotic Microangiopathy. The New England Journal of Medicine, 371, 654-666. https://doi.org/10.1056/NEJMra1312353

[4] Garg, A.X., Suri, R.S., Barrowman, N., et al. (2003) Long-Term Renal Prognosis of Diarrhea-Associated Hemolytic Uremic Syndrome: A Systematic Review, Meta-Analysis, and Meta-Regression. JAMA, 290, 1360-1370. https://doi.org/10.1001/jama.290.10.1360

[5] Moake, J.L. (2002) Thrombotic Microangiopathies. The New England Journal of Medicine, 347, 589-602. https://doi.org/10.1056/NEJMra020528

[6] Tran, P.N. (2018) Cobalmin Deficiency Presenting with Thrombotic Microangipathy (TMA) Features: A Systematic Review. Transfusion and Apheresis Science, 57, 102-106. https://doi.org/10.1016/j.transci.2018.01.003

[7] Booth, K.K., et al. (2011) Systemic Infections Mimicking Thrombotic Thrombocytopenic Purpura. American Journal of Hematology, 86, 743-751. https://doi.org/10.1002/ajh.22091

[8] Beckford, R. and Shah, G. (2010) Thrombotic Thrombocytopenic Purpura: A Review of the Disease Entity, Its Clinical and Laboratory Features, and Management Strategies. The Medicine Forum, 12, 12-15. https://doi.org/10.29046/TMF.012.1.005

[9] Blombery, P. and Scully, M. (2014) Management of Thrombotic Thrombocytopenic Purpura: Current Perspectives. Journal of Blood Medicine, 5, 15-23. https://doi.org/10.2147/JBM.S46458

[10] Wu, H.M., et al. (2013) Pulmonary Manifestations in Patients with Thrombotic Thrombocytopenic Purpura at a Community Hospital Setting. Chest, 144, 365A. https://doi.org/10.1378/chest.1703532

[11] George, J.N., et al. (2008) Lessons Learned from the Oklahoma Thrombotic Thrombocytopenic Purpura-Hemolytic Uremic Syndrome Registry. Journal of Clinical 
Apheresis, 23, 129-137. https://doi.org/10.1002/jca.20169

[12] Jamme, M. and Rondeau, E. (2017) The PLASMIC Score for Thrombotic Thrombocytopenic Purpura. The Lancet Haematology, 4, e148-e149. https://doi.org/10.1016/S2352-3026(17)30024-8

[13] Tsai, H.-M. (2006) Current Concepts in Thrombotic Thrombocytopenic Purpura. Annual Review of Medicine, 57, 419-436. https://doi.org/10.1146/annurev.med.57.061804.084505

[14] Rock, G.A., et al. (1991) Comparison of Plasma Exchange with Plasma Infusion in the Treatment of Thrombotic Thrombocytopenic Purpura. New England Journal of Medicine, 325, 393-397. https://doi.org/10.1056/NEJM199108083250604

[15] George, G.N. (2006) Thrombotic Thrombocytopenic Purpura. New England Journal of Medicine, 364, 1927-1936. https://doi.org/10.1056/NEJMcp053024

[16] Hovinga, J.A.K., et al. (2009) Survival and Relapse in Patients with Thrombotic Thrombocytopenic Purpura. Blood, 115, 1500-1511. https://doi.org/10.1182/blood-2009-09-243790

[17] Westwood, J.-P., et al. (2017) Rituximab Prophylaxis to Prevent Thrombotic Thrombocytopenic Purpura Relapse: Outcome and Evaluation of Dosing Regimens. Blood Advances, 1, 1159-1166. https://doi.org/10.1182/bloodadvances.2017008268

[18] Lee, W.R. (1983) Hemorrhagic Colitis Associated with a Rare Escherichia coli Serotype. The New England Journal of Medicine, 308, 681-685. https://doi.org/10.1056/NEJM198303243081203

[19] Karpman, D., et al. (2017) Haemolytic Uraemic Syndrome. Journal of Internal Medicine, 281, 123-148. https://doi.org/10.1111/joim.12546

[20] Tarr, P.I., et al. (2005) Shiga-Toxin-Producing Escherichia coli and Haemolytic Uraemic Syndrome. The Lancet, 365, 1073-1085. https://doi.org/10.1016/S0140-6736(05)71144-2

[21] Fakhouri, F., et al. (2017) Haemolytic Uraemic Syndrome. The Lancet, 390, 681-696. https://doi.org/10.1016/S0140-6736(17)30062-4

[22] Hossain, M.A., et al. (2018) Atypical Hemolytic Uremic Syndrome: Laboratory Characteristics, Complement-Amplifying Conditions, Renal Biopsy, and Genetic Mutations. Saudi Journal of Kidney Diseases and Transplantation, 29, 276-283. https://doi.org/10.4103/1319-2442.229287

[23] Nickavar, A. and Sotoudeh, K. (2013) Assessment, Treatment and Prevention of Atypical Hemolytic Uremic Syndrome. International Journal of Preventive Medicine, 4, 6-14.

[24] Phillips, E.H., Westwood, J.P., Brocklebank, V., et al. (2016) The Role of ADAMTS-13 Activity and Complement Mutational Analysis in Differentiating Acute Thrombotic Microangiopathies. Journal of Thrombosis and Haemostasis, 14, 175-185. https://doi.org/10.1111/jth.13189

[25] Loirat, C. and Frémeaux-Bacchi, V. (2011) Atypical Hemolytic Uremic Syndrome. Orphanet Journal of Rare Diseases, 6, 60. https://doi.org/10.1186/1750-1172-6-60

[26] Martinez-Barricarte, R., Pianetti, G., Gautard, R., et al. (2008) The Complement Factor H R1210C Mutation Is Associated with Atypical Hemolytic Uremic Syndrome. Journal of the American Society of Nephrology, 19, 639-646. https://doi.org/10.1681/ASN.2007080923

[27] Raina, R., Krishnappa, V., Blaha, T., Kann, T., Hein, W., Burke, L. and Bagga, A. (2019) Atypical Hemolytic-Uremic Syndrome: An Update on Pathophysiology, Diagnosis, and Treatment. Therapeutic Apheresis and Dialysis, 23, 4-21. 
https://doi.org/10.1111/1744-9987.12763

[28] Parente, R., Clark, S.J., Inforzato, A. and Day, A.J. (2016) Complement Factor H in Host Defense and Immune Evasion. Cellular and Molecular Life Sciences, 74, 1605-1624. https://doi.org/10.1007/s00018-016-2418-4

[29] Noris, M. and Remuzzi, G. (2013) Overview of Complement Activation and Regulation. Seminars in Nephrology, 33, 479-492. https://doi.org/10.1016/j.semnephrol.2013.08.001

[30] Asif, A., Vachharajani, T., Salman, L. and Nayer, A. (2014) A Simplified Approach to the Diagnosis of Atypical HUS: Clinical Considerations and Practical Implication. The Open Urology \& Nephrology Journal, 7, 91-94. https://doi.org/10.2174/1874303X01407010091

[31] Salvadori, M. and Bertoni, E. (2013) Update on Hemolytic Uremic Syndrome: Diagnostic and Therapeutic Recommendations. World Journal of Nephrology, 2, 56-76. https://doi.org/10.5527/wjn.v2.i3.56

[32] Nayer, A. and Asif, A. (2016) Atypical Hemolytic-Uremic Syndrome: A Clinical Review. American Journal of Therapeutics, 23, e151-e158.

https://doi.org/10.1097/MJT.0b013e31829b59dc 